\title{
Part-Structure Modifiers
}

Friederike Moltmann

CUNY, Graduate Center

\section{Part-structure modification as a linguistic phenomenon}

The aim of this paper is to show that a particular group of expressions, which have not been recognized as such before, form a uniform class in their syntactic distribution and their general semantic function. These expressions include as $a$ group, as a collection, as individuals, as a whole, together, alone, individual(ly), separate(ly), entire(ly), and whole (wholly). The semantic function of such expressions in the various contexts in which they occur always is to modify the part structure of an entity. Hence the expressions are appropriately called part-structure modifiers.

The syntactic characteristic shared by all part-structure modifiers is that they occur both in adnominal and in adverbial position, that is, they may modify both NPs and VPs. In adnominal position, part-structure modifiers occur either postnominally as in $(1 \mathrm{a}, \mathrm{b})$ or prenominally as adjectival modifiers, as in (1c, d): 1

(1) a. The boxes together / as a whole / as a group are heavy.

b. The box alone is heavy.

c. The individual boxes are heavy.

d. The whole / entire class passed the exam.

The same part-structure modifiers (possibly with the adverbial suffix $-l y$ ) occur in adverbial position, as in (2):

(2) a. John and Mary worked on the problem together / as a group / alone I individually / separately.

b. John forgot the poem entirely / wholly.

The challenge for a semantic analysis of part-structure modifiers resides in the fact that part-structure modifiers typically exhibit various different readings in the two syntactic positions and in different semantic contexts. My goal in this paper is to give an outline of a unified analysis of part-structure modifiers which derives the various readings they exhibit on the basis of the same underlying meaning, taking into account the syntactic and semantic context in which the part-structure modifiers occur. This paper can give only a sketch of how a unified semantic analysis of partstructure modifiers might look like. There is still a lot of empirical material about part-structure modifiers to be uncovered and many subtle differences among readings of different part-structure modifiers to be explained.

The most important point of this paper is that part-structure modification is a phenomenon of its own and distinct from more familiar linguistic phenomena. In particular, it has to be distinguished from four constructions which share similarities with part-structure modifiers in either syntactic or semantic respects: quantification, restrictive modification, expressions of completion such as complete(ly) and part(ly), and binary distributive event quantifiers such as one at a time. At the end of this paper, I will compare in more detail these constructions with part-structure modification, showing that despite semantic similarities or identical 
semantic effects in some cases, part-structure modifiers differ significantly from those constructions in their underlying semantics.

The analysis of part-structure modifiers that I will give in this paper is part of the more general account of part-structure-related expressions in terms of situated part structures in Moltmann (to appear). The present paper goes beyond that work, however, in spelling out in more detail the analysis of part-structure modifiers and in focusing on the distinctive nature of part-structure modification as a linguistic phenomenon.

\section{The readings of adnominal and adverbial part-structure modifiers}

Part-structure modifiers, in quite systematic ways, exhibit different readings in adnominal and adverbial position. The most important generalizations about the readings that part-structure modifiers exhibit in the two positions are as follows.

In adnominal position, part-structure modifiers exhibit readings which are basically independent of the content of the predicate. In this position, the main function of part-structure modifiers is to influence the way the predicate relates to the parts and the whole of the argument. Thus, the sole function of together in (3) is to enforce a collective reading of the predicate, the sole function of alone in (4) (on one reading) to enforce a 'nonpartitive' reading of the predicate, and the sole function of individual in (5) and separate in (6) to enforce a distributive reading of the predicate; one function of whole and entire in (7) is to allow for a distributive reading that would otherwise not be available:

(3) The boxes together are heavy.

(4) The box alone is heavy.

(5) a. I liked the individual paintings (but not the exhibition as a whole).

b. I cannot remember the individual students (but only the class as a whole).

(6) John put the two suits in separate suitcases.

(7) a. The whole / entire collection is expensive.

b. John gave the whole / entire class an A.

There are a few other readings that part-structure modifiers in adnominal position may exhibit. I will largely disregard such readings in what follows; but for completeness' sake, they should be mentioned nonetheless.

Alone displays an additional reading on which it is equivalent to only, as in

(8) John alone passed the exam.

Moreover, adnominal part structure modifiers may allow for readings related to the content of the predicate when they are focused:

(9) a. John alóne solved the problem.

b. John and Mary togéther solved the problem.

Under focusing, alone in (9a) may mean 'without the help of others' and together in (9b) may specify John and Mary as coagents of the event of solving the problem.

Adverbial part-structure modifiers differ from adnominal part-structure modifiers in that they generally exhibit readings that are, in some way or other, dependent on the content of the predicate. As was pointed out by Lasersohn (1990) (in regard to together), part-structure modifiers exhibit basically two kinds of readings: action-related and space-time related readings. 
On the action-related reading, adverbial together when relating to an agentive subject may display either a group-action reading as in (10a) or a cooperated-action reading, as in (10b):

(10) a. John and Mary solved the problem together.

b. John and Mary thought about the problem together.

Of course, corresponding readings are possible also with other thematic roles than agenthood, for example in (11):

(11) a. The two books fell on the floor together.

b. John showed Bill and Mary the exhibition together.

To simplify the discussion, though, I will restrict myself to part-structure modifiers applying to agents.

Adverbial alone, when relating to an agentive subject, displays corresponding readings. It may display either a sole-agent reading, as in (12a), or an isolatedaction reading, as in (12b):

(12) a. John solved the problem alone.

b. John thought about the problem alone.

Individual and separately display more or less equivalent readings in adverbial position. Both specify separated actions when modifing an action predicate:

(13) a. The students solved the problem individually / separately.

b. John greeted the people individually / separately.

Also wholly and entirely display action- or event-related readings. Thus, in (14), they yield what one may call a 'complete-involvement reading':

(14) The clouds have wholly / entirely disappeared.

When modifying other predicates, in particular those specifying spatio-temporal locations, part-structure modifiers display space-time related readings. Thus, together in (15a) specifies that John and Mary are spatially and temporally close, alone in (15b) that John is isolated in time and space, separately and individually in (15c) that the cups are separated in space, and entirely in (15d) that the guests cover a spatial location:

(15) a. John and Mary sat together.

b. John sat alone.

c. The cups are standing separately / ? individually on the cupboard.

d. The guests filled the room entirely.

There are systematic correlations between semantic predicate types and readings of adverbial part-structure modifiers. In general, when the predicate describes an action, adverbial part-structure modifiers yield readings that involve the relation of coagenthood or cooperation during the action. When the predicate describes a spatio-temporal location, then adverbial part-structure modifiers yield readings that involve the relation of spatio-temporal closeness.

What is important about the various readings that adverbial part-structure modifiers display is that they are not generally all available for a given occurrence of the modifier. Rather only some of the readings are available in a given semantic 
context. Thus, in (10a), only a group-action reading, not a spatio-temporal proximity reading, is available, and in (15a) only a spatio-temporal proximity reading. This clearly shows that the different readings of adverbial part-structure modifiers are not a matter of an ambiguity, but rather of context-dependency. Partstructure modifiers must involve some more general, abstract meaning, which, in a given context, will yield the relevant reading. Also, of course, this meaning should be the same that part-structure modifiers have in adnominal position. This then defines two challenges for a semantic analysis of part-structure modifiers. First, the analysis should provide a uniform meaning of part-structure modifiers in adverbial and adnominal position. Second, the analysis should derive the various readings of part-structure modifiers from the syntactic and semantic context in which the modifiers occur. The analysis given in what follows aims to meet these challenges.

\section{The basic idea}

\subsection{The general meaning of part-structure modifiers}

The basic idea is this. Part-structure modifiers express general properties of part structures of entities in situations. The different readings of part-structure modifiers then arise because part-structure modifiers apply to different situations. Such situations differ in their information contents, which in turn correspond to different ways of satisfying part-structure properties, yielding different readings of partstructure modifiers when applying to such situations. Given this very general description of the account, three basic questions have to be answered:

[1] What are part structures?

[2] What are situations? And what situations do part-structure modifiers apply to?

[3] What are part structures in situations?

Concerning the first question, an important assumption of the account is that certain entities, in particular groups (plural referents), may have different part structures in different situations. One way in which this can happen is by a group being divided differently into parts (group members or subgroups) in different situations. But there are also other ways for an entity to have different part structures in different situations; and they have to do with the particular way part structures are conceived. On one view of part structures (cf. Simons 1987, Moltmann, to appear), part structures not only specify what the parts of an entity are, but also whether an entity or its parts are integrated wholes. Being an integrated whole or having integrity distinguishes, for example, the referent of the sand and the referent of the heap of sand. These entities have the same parts, but they differ in integrity (in the relevant situation). But integrity does not always individuate different entities. One and the same entity may be an integrated whole in one situation, but not in another situation. For example, one and the same entity may be characterized as an integrated whole in one situation by referring to it as the collection of books and as not being an integrated whole in another situation by referring to it as the books.

Concerning the second question, the only important assumption about situations in the present context is that situations are partial specifications of entities with properties. On the view that I will adopt, they are primitives and they form a parameter relative to which every expression in a sentence will be evaluated. Situations in fact can be identified with functions mappping an expression to an extension. Unlike possible worlds, situations yield only partial functions. A sentence, for example, need not be evaluated as either true (1) or false (0) relative to a situation $s$; it may also be evaluated as undefined (\#) relative to $s$. Another important assumption about situations is that they have a domain. Such a domain is 
a set of entities which may consist of only some of the entities there are in the universe. Only those entities should be in the domain of a situation which the situation specifies for some property positively or negatively.

These two properties of situations, partial specification of entities with properties and association with a partial domain of entities, provide the answer to the third question. The partiality of the domain of a situation allows a situation to contain only some and not all of the actual parts of an entity. For example, in the case of a group, the domain of the situation may contain only some of the subgroups. The second property implies that situations may specify or fail to specify an entity or its parts as integrated wholes and in this way yield different part structures of the same entity.

An important notion for part structures and in particular for the semantic analysis of part-structure modifiers is the notion of integrated whole. The notion of integrated whole is a difficult one (see the recent discussion in Simons 1987). However, for the analysis of part-structure modifiers, a rather simple notion of integrated whole suffices, namely the notion of $R$-integrated whole (cf. Simons 1987). An entity is an $R$-integrated whole just in case all its parts are connected by some appropriate relation $R$ (or rather its transitive closure $R$ trans) and no part of it is connected by $R$ (or rather $R$ trans) to anything that is not a part:

(16) For an appropriate relation $R$, $x$ is an $R$-integrated whole in a situation $s$ iff for all $x^{\prime}, x^{\prime \prime}, x^{\prime}<s, x^{\prime \prime}<s x$, $R_{\text {trans }}\left(x^{\prime}, x^{\prime}\right)$, and for no $x^{\prime}, y, x^{\prime}<_{s} x, \neg y<_{s} x, R_{\operatorname{trans}}\left(x^{\prime}, y\right)$.

A crucial issue concerning the notion of $R$-integrated whole is: what counts as an appropriate relation? An appropriate relation is, for example, closeness in space or time, but not distance. Moreover, only only nonlogical relations are appropriate. Conditions on appropriateness play an important role in the semantics of partstructure modifiers, as we will see, and there will be more conditions to be imposed on what counts as an appropriate relation.

\subsection{The source of the difference between adnominal and adverbial part-structure modifiers}

On my account, the source of the difference among the readings of adnominal and adverbial part-structure modifiers resides in the fact that part-structure modifiers in adnominal position are evaluated with respect to a different kind of situation than part-structure modifiers in adverbial position. What exactly are those situations?

Adnominal part-part structure modifiers apply to a situation which is associated with the NP only, a situation, roughly speaking, with respect to which the speaker intends the descriptive content of the NP to be evaluated. Thus, when uttering the box alone, the speaker has in mind a particular situation in which there is exactly one box and alone will modify the box in that situation. I will call the situation associated with the utterance of an NP the reference situation of that NP.

Adverbial part-structure modifiers, by contrast, relate to the situations that the evaluation of the predicate is based on, the situations that make up the proposition the sentence expresses. Following Situation Semantics, these situations can be called the described situations. Adverbial part-structure modifiers then, more precisely, are evaluated with respect to certain subsituations of the described situations, namely those subsituations that only contain information about the described event.

The crucial difference between reference situations and described situations in the present context is that, unlike described situations, reference situations generally 
do not have access to the information provided by the predicate. This is simply an instance of the following more general principle about the evaluation of predicates and arguments:

(17) Predicate-Argument Asymmetry Information provided by a predicate is inaccessible for the evaluation of the arguments.

As a consequence, adnominal part-structure modifiers in general cannot have readings that relate specifically to the content of the predicate, in particular, the kind of action or event described by the predicate. By contrast, adverbial part-structure modifiers apply to situations whose information content consists just in information about the described event, as provided by the predicate.

Before developing this account in any detail, some further general assumptions have to be spelled out concerning the status of reference situations and the treatment of distributivity.

\subsection{Further general assumptions}

\subsubsection{Reference situations}

One important assumption concerns the status of reference situations. Part-structure modifiers as well as certain other part-structure-related phenomena (cf. Moltmann, to appear) motivate a new conception of the values of referential NPs and of arguments of predicates. On this view, referential NPs do not just refer to entities, and predicates do not just take entities as arguments. Rather, referential NPs refer to pairs consisting of an entity and a reference situation. Correspondingly, predicates do not just take entities as arguments, but rather pairs consisting of an entity and a reference situation. The function of the reference situation as part of an argument of a predicate, basically, is to convey what the relevant part structure of the entity is. This part structure may be important for the evaluation of a part-structure-sensitive predicate, for example distinguish as in (18):

(18) John cannot distinguish the students.

Distinguish is sensitive to the part structure of an argument because a sentence such as (18) in which distinguish has a plural argument can be understood in a variety of different ways, depending on the part structure of the group of students. (18) may mean, for example, that John cannot distinguish any one student from any other student or that John cannot distinguish one relevant subgroup of students from the other relevant subgroup of students. Thus, distinguish in (18) does not just apply to the group $x$ of students, but rather to a pair consisting of $x$ and a situation $s$, where $s$ will give information about what the relevant parts of $x$ are (whether they are individual members or certain subgroups) and hence what exactly John is unable to distinguish. The truth conditions of (18) can thus be paraphrased roughly as in (19), where $s^{\prime}$ is a reference situation, $s$ a described situation, and sum an operation of group or sum formation (mapping the extension of students in $s^{\prime}$, the set of groups of relevant students in $s^{\prime}$, to the maximal group of students in $s^{\prime}$ ):

(19) [distinguish $]^{S}\left(\mathrm{John},<s u m\left(\left[\right.\right.\right.$ students $\left.\left.^{S}\right), s^{\prime}>\right)=1$ iff

John distinguishes in $s$ each part of $\operatorname{sum}\left([\text { students }]^{S}\right)$ in $s^{\prime}$ from any other part of $\operatorname{sum}\left([\text { students }]^{S}\right)$ in $s^{\prime}$. 
It is not the case, though, that only certain predicates are sensitive to the part structure of an entity in a situation and that hence only those predicates (relative to the relevant argument position) should take situations as part of their arguments. Rather all predicates with respect to all argument positions must be sensitive to situations. The reason is that every predicate allows for distributive readings with respect to each of its argument positions, and, given the particular view of distibutivity that I endorse, distributivity is sensitive to the part structure of an entity in the reference situation. Let me turn to this treatment of distributivity.

\subsubsection{The treatment of distributivity}

The treatment of distributivity that I endorse and that is discussed in more detail in Moltmann (to appear) rests on the assumption that predicates are neutral with respect to collective and distributive readings. One motivation for this assumption comes from the possibility of conjunctions of distributive and collective modifiers as in (20):

(20) Yesterday, John and Mary lifted the box individually and together.

Such conjunctions of distributive and collective modifiers present problems for the alternative views of distributivity, namely [1] the view that verbs are ambiguous between a collective and a distributive reading and [2] the view that distributivity is not part of the lexical meaning of a verb, but rather a matter of an implicit distributivity operator attaching either to the NP or to the VP. A conjunction of distributive and collective modifiers clearly is incompatible with the verb having either a collective or a distributive reading. Also it is incompatible with the exclusive choice of a distributive operator being either present or absent. Rather, such modifiers require that the verb have a neutral meaning as regards the distributivecollective distinction. Then, (20) simply means that John and Mary were involved in a complex event of lifting the box, part of which involved John and Mary individually and part of which involved John and Mary collectively. 2

One cannot content oneself, though, simply saying that predicates are neutral with respect to distributive and collective readings. The lexical meaning of a predicate clearly must specify in which way a group participates in the described event. If the concept 'lift the box' applies to John and Mary as a group, then we have to know who is the agent of the piano lifting or its subevents, John and Mary as a group, or John as an individual in one subevent and Mary as an individual in another subevent. In the first case, lifted the box applies to John and Mary as a group literally; in the second case, it applies distributively. It cannot apply in the same way in both cases. But this means that the neutrality of verbs with respect to the distributive-collective distinction has to be construed as a disjunction of two meanings: the literal collective meaning and the distributive meaning (or, in the case of a verb with more than one argument position, a collective meaning and several distributive meanings). The distributive meaning essentially consists in universal quantification over the relevant parts of the argument and application of the literal meaning to those parts.

The distributive disjunct in such a disjunctive meaning makes crucial reference to the reference situation, since it involves a quantifier ranging over the parts of the group in the reference situation. Thus, formally, heavy will have the following disjunctive meaning:

$$
\text { (21) }[\text { heavy }]^{S}=\lambda x s^{\prime}\left[[\text { heavylit }]^{S}(x)=1 \mathrm{v} \forall x^{\prime}\left(x^{\prime}<s^{\prime} x \rightarrow[\text { heavylit }]^{S}\left(x^{\prime}\right)=1\right)\right]
$$


Every verb allows for distributive readings with respect to every argument position and therefore involves reference situations for all of its argument positions. Consider (22):

(22) The women showed the men the children.

(22) allows for readings in which the event involves the women collectively or individually, the men collectively or individually, or the children collectively or individually; and it allows for any combination of such readings (that is, we will get $2 \times 2 \times 2$ readings). Thus, the lexical meaning of a verb requires distributive disjuncts for every argument position (and, thus, any $n$-place verb will require $2^{n}$ disjuncts).

Having laid out the general view of part structures, the assumptions about reference situations and distributivity, and the kinds of situations that adnominal and adverbial part structures apply, I can now turn to deriving the various readings that different adnominal and adverbial part-structure modifiers display.

\section{Deriving the readings of adnominal and adverbial together and alone}

\subsection{Adnominal together and alone}

The most straightforward cases of part-structure modifiers, which I have discussed in greater detail in Moltmann (1994), are together and alone. Let me review the essentials of that analysis. The general meaning of together is the property which holds of an entity $x$ in a situation $s$ just in case $x$ is an integrated whole in $s:$

\section{(23) $[$ together $]=[\mathrm{INT}-\mathrm{WH}(x, s)]$}

This general meaning suffices for deriving the various readings of adverbial and adnominal together.

Adnominal together specifies an entity as an integrated whole in the reference situation. However, here integrity will be required only in a rather 'abstract' sense, no particular relation $R$ being specified. But still, doing this, adnominal together yields a specific semantic affect, namely it prevents a predicate applying distributively to an argument. This prevention of a distributive reading is due to a general principle which I have justified in greater detail elsewhere (cf. Moltmann, to appear), namely the Accessibility Requirement. This requirement says that a predicate or reading of a predicate making reference to the parts of an argument can apply to an argument only if that argument is not an integrated whole in the reference situation:

\section{(24) The Accessibility Requirement}

A predicate or reading of a predicate making reference to the parts of an argument can apply to an entity $x$ in a situation $s$ only if $x$ is not an integrated whole in $s$.

The distributive disjunct of any predicate clearly makes reference to the parts of an argument and hence is subject to the Accessibility Requirement. Thus, when the boxes together in (3) refers to an entity $x$ in a reference situation $s, x$ will be specified as an integrated whole in $s$. Hence the distributive disjunct of heavy cannot apply to $x$ in $s$; only the collective disjunct can.

Alone has as its general meaning the property that holds of an entity $x$ in a situation $s$ if $x$ is not part of an integrated whole in $s$ : 


$$
[\text { alone }]^{S}=\lambda x[\neg \exists y(x<s y \& \text { INT-WH }(x, s))]
$$

Thus, adnominal alone specifies an entity as not being part of an integrated whole in the reference situation. The semantic effect of this is simply to prevent a marginal 'partitive reading'. Thus, in the case of the box alone in (4) the box will be specified in the reference situation not being part of an integrated whole, and hence, for example, it might not be viewed as part of a complex object, the box together with its content. As a result, the predicate could not possibly apply to the box in a kind of partitive way, by targeting the more complex object having the box as a part. In order for this explanation to work, though, it has to be considered a marginal option for a predicate to be applicable to an object $x$ in a situation $s$ just in case the predicate is true 'literally' of an object $y$ in $s$, where $x<_{s} y$ and INT$\mathrm{WH}(y, s)$. It is such an application that is excluded by the presence of alone.

\subsection{Adverbial together}

The readings of adverbial together and alone are based on exactly the same general meanings given in (23) and (25). But with these meanings, adverbial together and alone apply to different situations. Adverbial part-structure modifiers apply to the maximal subsituation $s e$ of any situation $s$ that the sentence might describe, where $s e$ contains only information about the event $e_{\text {. }} s_{e}$ can be defined as the maximal subsituation of $s$ which specifies an $n$-place predicate with respect to an $n$-tuple of entities positively or negatively only if one of these entities is the event $e$ itself:

(26) For a situation $s, s_{e}$

$=$ the maximal subsituation of $s$ such that for any predicate $P$,

$[P]^{S}\left(x_{1}, \ldots, x_{n}\right)=1$ or 0 , then $x_{i}=e$ or $x_{i}=e^{\prime}$ for some $i \in\{1, \ldots, n\}$, $e^{\prime}<e$.

Consider (10a) and suppose that $e=$ the event of John and Mary solving the problem. Then together specifies that John and Mary form an integrated whole in the maximal subsitation of any described situation that is about $e$. But this means that John and Mary must form an $R$-integrated whole for some relevant relation $R$ involving $e$ as an argument. And, given that there are no other entities in $s_{e}$ than John and Mary (besides $e$ itself), we get the following equivalence:

(27) [together] $S e$ (John and Mary) $=1$ iff for an appropriate relation $R$, $R^{S}(e, \mathrm{John}, \mathrm{Mary})=1$.

One such relation $R$ is the relation of being coagents of $e$. In this case, if together holds of John and Mary as a group, we have $\operatorname{COAGENT}^{S}(e, \mathrm{John}, \mathrm{Mary})=1$. But this is not the only relation that would be appropriate. Another appropriate relation would be one in which John and Mary just cooperate when doing $e$.

Now consider (15a) and suppose $e=$ the event of John and Mary sitting. Here $e$ basically consists just in a space-time region. An appropriate relation $R$ connecting John and Mary then is simply the relation BE AT that holds among $e$, John, and Mary if John and Mary both are at $e$. Crucial here is that $e$ must be connected in space and time. In this case, we have:

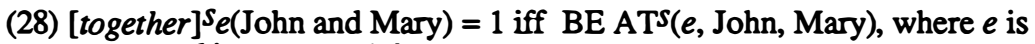
connected in space and time. 
Given this general account, one crucial question has be answered, namely what are appropriate relations involving an event? Why can one choose cooperation, but not lack of cooperation, and closeness in space and time, but not distance? Apparently, only those relations are appropriate that involve an event that is an integrated whole. An event that is an integrated whole is, for example, a single action, a coordinated action, or a state that is connected in space and time, but not a group of disconnected actions or a disconnected spatio-temporal location. Thus, we have the following condition on appropriate relations $R$ defining $R$-integrity:

(29) A relation $R_{e}$ is appropriate only if $\mathrm{NTT}-\mathrm{WH}(e)$.

The readings of adverbial alone can be derived in an exactly parallel way. Adverbial alone specifies failure of forming part of an integrated whole in the situation $s e$. This can be the case, for example, by virtue of lacking coagenthood with others or failing to be spatially and temporally close to others.

Let us now turn to the other part-structure modifiers, whose semantics can be given in an exactly parallel fashion.

\section{Deriving the readings of other part-structure modifiers}

\subsection{Individual(ly)}

It is natural to take individually and individual to be instances of the same lexical word having one and the same lexical meaning (their difference being only a matter of morphology). What is the lexical meaning of individual(ly)? Obviously, individually emphasizes that the individual members of the group are relevant parts of the group. Moreover, it excludes that subgroups of the group are relevant parts. Finally, it specifies that the entity it applies to is not an integrated whole; thus excluding its application to collective singular count NPs.

It is not a completely trivial matter to formulate these conditions correctly. A first approximation to the meaning of individual (ly) is the following property:

(30) the property that an entity $x$ has in a situation $s$ iff $x$ is not an integrated whole in $s$ and consists of parts that are integrated wholes in $s$ and no subgroup of such parts is a part of $x$ in $s$

Formally, this corresponds to:

$$
\begin{aligned}
& {[\text { individual }(l y)]^{s}=\lambda x[\neg \mathrm{INT}-\mathrm{WH}(x, s) \&(\forall y(y<s x \rightarrow \mathrm{INT}-\mathrm{WH}(y, s)) \&} \\
& \neg \exists Y(Y \subseteq\{y \mid y<s x\} \&|Y|>1 \& \operatorname{INT}-\mathrm{WH}(\operatorname{sum}(Y), s))]
\end{aligned}
$$

(31) requires that if individual(ly) holds of an entity $x$, then proper groups of parts of $x$ that are integrated wholes should not be integrated wholes. Given the way part structures are conceived (cf. Appendix), this implies (30).

However, there are problems with (31). (31) does not exclude that a group $x$ consists of parts in a situation $s$ that are subgroups and integrated wholes (though no sum of such subgroups will be a part of $x$ in $s$ ). However, the part structure of $x$ in $s$ should consist only of the individual members of $x$. In order to account for this, we have to make use of the notion of essential integrated whole. Only the individual members of a plural referent are essential integrated wholes. For subgroups, it is not essential whether they have integrity or not; they could only be 
accidental integrated wholes. The notion of essential integrated whole can be represented by 'INT-WH' taken as a one-place predicate so that 'INT-WH(y)' means ' $y$ is an essential integrated whole'. Then, the proper formulation of the meaning of individual(ly) is as follows:

(32)

$$
\begin{aligned}
& {[\text { individual }(l y)]^{s}=\lambda x[\neg \mathrm{INT}-\mathrm{WH}(x, s) \&(\forall y(\mathrm{y}<s x \rightarrow \mathrm{INT}-\mathrm{WH}(y)) \&} \\
& \neg \exists Y(Y \subseteq\{y \mid y<s x\} \&|Y|>1 \& \mathrm{INT}-\mathrm{WH}(\operatorname{sum}(Y), s))]
\end{aligned}
$$

Adnominal individual has basically either of two semantic effects. The first one is to enforce a strictly distributive interpretation as in (5). Strict distributivity means that the predicate must apply to all the individual group members and may not just distribute down to subgroups. This effect clearly follows from the way distributivity is conceived and from the kind of part structure that individual(ly) specifies. All and only the individual group members will be the situated parts of the group, and thus the universal quantifier in the distributive disjunct in the predicate's meaning will range only over those parts.

But why, with the predicates in $(5 \mathrm{a}, \mathrm{b})$, does distributivity have to apply? The reason is that there would be no other way for individual $(l y)$ to have any semantic effect and be relevant to the application of the predicate. The only way predicates such as like and remember to take into account the particular part structure specified by individual(ty) is to apply distributively (see Moltmann 1994, to appear).

In this respect, such predicates differ from predicates whose 'literal' lexical meaning is sensitive to the part structure of an argument. Examples of such predicates are distinguish, compare, rank, and list. With those predicates, individual has a different semantic effect, namely it enforces a particular reading of the predicate applying nondistributively. Compare (33a) with (33b):

(33) a. John cannot distinguish the individual students.

b. John cannot distinguish the students.

(33b) has a reading involving subgroups, for instance if John cannot distinguish the MIT students from the Harvard students. But (33a) lacks such a reading. It can only be true if John cannot distinguish any one student from any other student.

Adverbial individually applies with the same meaning to the situation $s e$ for $e$ being the described event. Given the notion of $R$-integrated whole, this means that individually prevents any proper subgroups to be integrated wholes with respect to a relation $R$ which involves the event $e$ as an integrated whole. Thus no two individual members of $x$ may stand in such a relation. But this means that no two individual members may stand in a relation of coagenthood or cooperation with respect to the described event, or may be spatially and temporally close (if the event is a state). Thus, individual(ly), when applying to an entity $x$ in a situation $s_{e}$, has the following implication:

(34) [individually] $S_{e}(x)=1$, then for any $x^{\prime}, x^{\prime \prime}, x^{\prime}<_{s} x, x^{\prime \prime}<_{s} x$, for no appropriate $R, R\left(e^{\prime}, x^{\prime}, x^{\prime}\right)=1$

It then only depends on the nature of $e$ what sort of relation individually specifies as not holding among members of $x$.

\subsection{Separate(ly)}


Separate(ly) has quite similar semantic effects in adnominal and adverbial position to individual(ly). Like individual, adnominal separate may enforce a distributive reading, as in (6). However, this effect is achieved on the basis of a rather different meaning than that of individual. Unlike individual, separate has a relational meaning. In this respect, it classifies together with expressions like different, neighboring, and parallel. The contrast can best be seen from the use of individual and separate with singular count nouns, as in (35):

(35) a. A separate / different container is necessary.

b. An individual mathematician could solve the problem.

With singular count nouns, separate, like other relational adjectives, requires an implicit argument to be filled in by the previous discourse. Thus, separate in (35a) means 'separated' from some other, previously mentioned container. By contrast, individual in (35b) is redundant, emphasizing the individuality of a single mathematician. Like other relational adjectives, separate, when modifying a singular count noun, involves a particular relation of the NP-referent to some previously mentioned entity, whereas, when modifying a plural noun, it expresses a property of a group whose members are connected by that same relation. In the case of separate(ly), this relation is the relation of being disconnected by some contextually relevant relation.

What is a contextually relevant relation? Given the notion of situation, there is a simple way of defining contextual relevance. Contextually relevant relations are simply those relations for which the relevant situation specifies some $n$-tuple of entities positively ot negatively. Thus, as the meaning of separate(ly), we have (36), again making use of essential integrity:

$$
\begin{aligned}
& {[\text { separate }(l y)]^{S}=\lambda x[\neg \mathrm{INT}-\mathrm{WH}(x, s) \& \forall y(y<s x \rightarrow \mathrm{INT}-\mathrm{WH}(y)) \&} \\
& \left.\forall x^{\prime} x^{\prime \prime}\left(x^{\prime}<_{s} x \& x^{\prime \prime}<_{s} x \& \rightarrow \exists R R^{s}\left(x^{\prime}, x^{\prime \prime}\right)=0\right)\right]
\end{aligned}
$$

Adnominal separate then specifies that all the parts of the relevant entity $x$ are disconnected by some relation in the reference situation $s$. The effect of this, generally, is that no group of such parts may be an integrated whole $s$, and thus be in the domain of the situation and a part of $x$. In order for separate, then, to have any semantic effect, it will enforce a distributive reading with relevant predicates.

Adverbial separately, again, applies to the situation $s_{e}$ for the described event $e$. Given the definition of $s_{e}$, this means that separately requires that the situated parts of the group be disconnected by some relevant relation involving the described event - or a part of it:

(37) [separately] ${ }^{S} e(x)=1$, then for an appropriate relation $R$, for some $e^{\prime}, e^{\prime}<e$, $R^{S}\left(e^{\prime}, x, x^{\prime \prime}\right)=0$ and $\mathrm{NNT}-\mathrm{WH}\left(e^{\prime}\right)$.

Separately requires disconnectedness among group members by some relation, whereas individually denies connectedness. In both cases, as a result, no subgroup will be specified as an integrated whole in the relevant situation, and this is what enforces the strictly distributive interpretation in both cases (for predicates not making reference to the part structure of an argument).

\subsection{Entire(ly) and whole (wholly)}


Adnominal entire andwhole have two kinds of semantic effects. The first effect is to allow for a distributive reading that might otherwise not be available. Examples for this were given in (7). The distributive reading that entire and whole trigger consists in that the predicate applies to every actual part of the entity. Thus, (7b) (on the relevant reading) implies that every student in the class got an A.

In $(7 \mathrm{a}, \mathrm{b})$, the presence of entire and whole is required for the distributive reading to be available, because otherwise there would be a violation of the Accessibility Requirement. Thus, entire and whole specify an entity as not being an integrated whole in the relevant situation. Moreover, they specify that every relevant part of the entity be in the reference situation. Thus, the following property can be taken as the lexical meaning of entire(ly) (and whole (wholly)):

(38) $[\text { entire }(l y)]^{S}=\lambda x\left[\neg\right.$ INT-WH $\left.(x, s) \& \forall x^{\prime}\left(x^{\prime}<x \rightarrow x^{\prime}<s x\right)\right]$

The second semantic effect that entire and whole may have in adnominal position arises with part-structure-sensitive predicates. The relevant kinds of readings are available in the following examples:

(39) a. The map covers the whole table.

b. John compared the whole class.

The semantic effect of whole in (39a) is not to trigger a distributive reading of the predicate, but rather to induce a particular reading of cover applied collectively, namely a reading on which every part of the table is covered by part of the map. Similarly, whole in (39b) does not yield a distributive reading, but rather allows for the part-structure sensitive predicate compare to apply to the class collectively.

(38) also accounts for adverbial entire(ly) and whole (wholly). Consider (14) and suppose $e=$ the event of the clouds' disappearing. Then entirely specifies that the clouds are not an integrated whole in $s e$ and every part of the group of clouds is present in $s_{e}$. The first condition is not relevant here; but the second condition is crucial. It means that every part of the group of clouds must stand in some relevant relation $R$ to $e$. Generally, the only relevant relation will be a thematic relation expressed by the verb. In the present case, this is the relation THEME, and so we have (40) (where THEME does not relate a single cloud to the entire event, but rather only to a part of it):

(40) [entirely] $]_{e}(x)$ iff for every $x^{\prime}<x$, for some $e^{\prime}<e, \operatorname{THEME}^{S}\left(x^{\prime}, e\right)$.

Thus, entirely requires 'complete distributive' participation of the relevant participant in the event.

\section{Part-structure modifiers and other constructions}

The result of this paper so far is that a significant class of expressions, 'partstructure modifiers', could be analysed in a uniform way as predicates of part structures of entities in situations. In the remainder of this paper, I want to contrast the phenomenon of part-structure modification with a number of related constructions which of ten lead to quite similar or even identical semantic contributions in a sentence. The emphasis will be on the underlying different semantics that distinguishes part-structure modifiers from those other constructions.

\subsection{Quantification}


Certain part-structure modifiers seem to have the same semantic effects as universal distributive quantifiers. ${ }^{2}$ Thus, individual in (41a) seems to be equivalent to floated each in (41 $\left.\mathrm{a}^{\prime}\right)$, and whole and entire in (41b) to partitive all in (41b'):

(41) a. The individual children were praised.

a'. The children each were praised.

b. The whole family was praised.

b'. All of the family was praised.

However, there are major difference between floated and partitive quantifiers and part-structure modifiers such as individual and whole. First, floated each does not allow for collective predicates holding of the entire entity, as in (42a, b); but such collective predicates make perfect sense with individual and whole, as was seen in (33a):

(42)\# The students each could not be distinguished.

Moreover, part-structure modification does not exhibit the characteristics of quantification, i.e. the ability to interact in scope with other quantifiers and the ability to bind variables. Thus, individually in (43a) cannot act, like each in (43b), as a universal distributive quantifier taking wide scope over exactly two presents:

(43) a. The individual children received exactly two presents.

a'. The children each received exactly two presents.

Similarly, individual in (44a) cannot act like the universal partitive quantifier each in (44b) taking wide scope over two students:

(44) a. Two students solved the individual problems.

b. Two students solved each of the problems.

The inability of part-structure modifiers to bind variables can be seen from the contrast between (45a) and (45b):

(45) a. The whole family drove its own car.

b. The family members each drove their own car.

(45a), unlike (45b), implies that there was only one car for the entire family, which means that whole in (45a) cannot, like each in (45b), act as a universal quantifier ranging over the members of the family and binding the pronoun its.

\subsection{Restrictive modification}

Part-structure modifiers generally occupy the same syntactic positions as restrictive (adnominal or adverbial) modifiers. But, semantically, part-structure modifiers have a very different function than restrictive modifiers.

The most difference is that restrictive modifiers do serve to influence the evaluation of the predicate. For example a collective relative clause, which clearly has a restrictive function, cannot have any influence on whether the predicate should apply collectively or distributively. Thus, the relative clause in (46) does not require a collective reading of the predicate:

(46) The boxes that are stacked on each other are too heavy. 
Even part-structure modifiers in relative clauses do not have any influence on the way the predicate applies. Thus, together in (47a) does not trigger a collective interpretation of the predicate and separately in (47b) does not trigger a distributive interpretation of the predicate:

(47) a. The boxes that belong together are too heavy.

b. The people who are standing separately in the room form a committee.

Adnominal restrictive modifiers only provide information that helps to identify the referent of the NP. By contrast, adnominal part-structure modifiers provide information which induces a perspective on the entity and serves to, in some way, influence the evaluation of the predicate.

\subsection{Expressions of completion}

Certain part-structure modifiers, whole, wholly, and entire(ly) at first sight seem to be synonymous with complete(ly) However, as it turns out, such synonymy appears only in particular contexts of occurrence. Completely has a rather different underlying semantics. It is not a part-structure modifier, but rather belongs to the category of 'expressions of completion' (cf. Molmann 1990, to appear), which has a semantics of its own. Other expressions of completion are, for example, halfway, partly, and partially. effect:

In (48a) and (48b), completely and entirely/wholly have the same semantic

(48) a. The clouds have completely disappeared.

b. The clouds have entirely / wholly disappeared.

But in other contexts complete(ly) and entire(ly)/whole (wholly) are not equivalent. Thus, complete is acceptable in (49a), but not in (49b), whereas entire and whole are hardly acceptable in (49a'), though they are fine in (49b'):

(49) a. the complete destruction of the city

b. \# the complete night

$a^{\prime}$. ?? the entire / whole destruction of the city

$b^{\prime}$. the entire / whole night

Complete, unlike entire and whole, can apply only to nouns that may denote abstract objects such as the destruction of the city (taken as an abstract event). The function of complete then is to specify that the concrete object referred to by the NP (in (49a), the concrete event of destroying the city) is 'complete' relative to that abstract object. Thus, complete has a relation as its meaning which holds between a concrete object $x$ and an abstract object $X$ iff every part of $X$ is instantiated (INST) by some part of $x$ :

\section{(50) $[\text { complete }(l y)]^{S}=\lambda x X\left[\forall X^{\prime}\left(X^{\prime}<X \rightarrow \exists x^{\prime}\left(x^{\prime}<x\right.\right.\right.$ \& INST $\left.\left.\left(x^{\prime}, X^{\prime}\right)\right)\right]$}

As an adverbial, completely applies with the same lexical meaning to the described event. It specifies that every part of the abstract event expressed by the event predicate is instantiated by some part of the concrete described event.

The underlying difference between completely as an expression of completion and entirely (wholly) as a part-structure modifier should manifest itself also in other 
contexts. One of them is this. As is discussed in Moltmann (1990), events may have more than one part structure. For example, an event of the paper being wrong may have a participant-related part structure (where the event parts correspond to the parts of the paper) and a degree-related part structure (where the event parts correspond to the degrees of being wrong). As a general fact, whenever an expression making reference to the part structure of an event applies to an event with $n$ different part structures, it yields $n$ different readings. This is because any part structure can be selected for the evaluation of the part-structure sensitive expression. Since completely applies directly to events (abstract and concrete events), it should allow for multiple readings corresponding to the number of part structures of the event. By contrast, entirely should allow for only exactly one reading, relating to the relevant participant that is modified. The data seem to speak in favor of that, though intuititions are not totally solid. Compare (51a) with (51b) and (52a) with (52b):

(51) a. This paper is completely wrong.

b. This paper is entirely wrong.

(52) a. The paint covered the photograph completely.

b. The paint covered the photograph entirely.

(51a) has two readings, one on which the paper as a whole is as wrong as it could possibly be, and one on which every part of the paper is wrong (in the normal sense). The first reading involves the part structure of the described event that correlates with the parts of the paper; the second reading involves the part structure of the described event that correlates with the degrees of being wrong. (51b), by contrast, seems to have only one reading, namely on which every part of the paper is wrong.

Similarly, (52a) has two readings: either the paint's coverage of the picture is opaque to the highest degree or every part of the picture is covered by paint. By contrast, (52b) tends to allow for only the latter, participant-related reading.

Thus, even though completely shares one reading with entirely in adverbial position, it has a different underlying semantics, which manifests itself in the greater range of readings completely exhibits and in the kinds of nouns complete may modify in adnominal position.

\subsection{Binary distributive event quantifiers}

Another type of expression semantically related to part-structure modifiers are binary distributive event quantifiers (cf. Moltmann 1991). Examples are one at $a$ time and in chapters, as in (53):

(53) a. John greeted the students one at a time.

b. John wrote the book in chapters.

Binary distributive event quantifiers always involve two quantifiers (hence the name): one ranging over the parts of the described event and one ranging over the parts of some event participant. Thus, one at a time ranges both over event parts (which corresponds to the at a time-part) and over parts of some participant. In the case of (53a), this is the event of greeting the students and the group of students. One at a time in (53a) then specifies that each temporal part of this event involves a different member of the group of students.

Some binary distributive event quantifiers yield effects quite similar to certain part-structure modifiers in adverbial position. Thus, one at a time in (53a) mean almost the same as that of individually in (54): 
(54) John greeted the students individually.

The contribution of individually in (54) also amounts to an association of event parts with different students. Why then should individually not be analysed as a binary distributive event quantifier in the first place, rather than a part-structure modifier? For one thing, of course, because of the interest in maintaining a uniform analysis of individual and individually. There is no way of treating the adjective individual as a binary distributive event quantifier. But there are also semantic differences between binary distributive quantifiers and part-structure modifiers. Binary distributive event quantifiers explicitly range over parts of the described event, and they may range over specified kinds of event parts. Thus, one at a time and individually range only over temporal parts of the event. By contrast, individually may relate to event parts only in an unspecific way; it could not possibly make reference to specific kinds of event parts.

\section{Summary}

The goal of this paper was to establish part-structure modification as a linguistic phenomenon of its own. Several generalizations were made concerning partstructure modifiers. First, all part-structure modifiers systematically occur in both adnominal and adverbial position. Second, all part-structure modifiers display readings related to the interpretation of the predicate in adnominal position and readings related to the described event in adverbial position

Also more general points have been established. The first one concerns the importance of the notion of a part structure in a situation. Part-structure modifiers, should they have a unified semantics, involve part structures that are relative to a situation. The different readings of adnominal and adverbial part structures come about precisely because different situations lead to different part structures.

The other general point concerns the notion of integrated whole. All partstructure modifiers, as far as they were considered in this paper, have a lexical meaning that involves the notion of integrated whole. Thus, part-structure modifiers, like a variety of other linguistic phenomena (cf. Moltmann to appear), emphasize the importance of the notion of integrity in natural language semantics.

\section{Appendix: Situated Part Structures}

This appendix serves to characterize the notion of situated part structure. Situated part structures have three components: a situation, an actual part structure and a situated part structure.

For the purpose of the semantics of part-structure-sensitive expressions, it is not important what axioms are assumed for actual part structures. Actual part structures may, for that matter, simply be taken as traditional mereological part structures with transitivity and closure under sum formation (though better not extensionality, i.e., entities are the same if they have the same parts):

\section{(1) Definition}

$(E,<)$ is an actual part structure iff
(i) $x<y \& y<z \rightarrow x<z$
(ii) $\neg x<x$
(iii) For any nonempty set $X \subseteq E$, $\operatorname{sum}(X)$ exists. 
A situated part structure in a situation $s$ then is composed of two part structures: an actual part structure and a part structure in a situation $s(\mathrm{D}(s),<s)$ (where $D(s)$, recall, was the domain of $s$ ). The situated part relation $<s$ can be considered a restriction of the actual part relation <. Unlike the latter, is is not generally transitive and closed under sum formation.

(2) Definition

$(s,(D(s),<s),(E,<))$ is a situated part structure iff $(E,<)$ is an actual part structure and (i)-(iv):

(i) $D(s) \subseteq E$

(ii) $<s \subseteq<$

(iii) $x<_{s} y \& y<_{s} z \& \neg$ INT-WH $(y, s) \rightarrow x<s z$

(iv) $X \subseteq D(s) \& \forall x(x \in X \rightarrow \operatorname{INT}(x, s)) \& \operatorname{sum}(X) \in \mathrm{D}(s) \rightarrow \operatorname{INT}(\operatorname{sum}(X), s)$

(2iv) says that a set $X$ of integrated wholes in a situation $s$ has a sum in $s$ only if that sum is itself an integrated whole in s. (2iv) thus limits sum formation to sets of entities that lack integrity. From (2iv), it can also be derived that subgroups of a group are not part of the group, unless they are themselves integrated wholes.

\section{Notes}

1 Standard sorts of syntactic evidence shows that part-structure modifiers appearing after an NP may form a constituent with the NP, rather than the VP:

(1) a. The two chairs together and the box alone weigh the same.

b. It was John alone who solved the problem.

c. The weight of the boxes together exceeds 100 pounds.

2 In fact, Schwarzschild (1994) takes the part-structure modifier together to be the counterpart of floated each. But, in fact, the counterparts of together are the partstructure modifiers individual and whole (entire), rather than the quantifier each.

\section{References}

Lasersohn, P. (1990): 'Group action and spatio-temporal proximity'. Linguistics and Philosophy 13.

Molmann, F. (1990): 'The multidimensional part structure of events'. WCCFL 9, Stanford Linguistics Student Association, Stanford University, Stanford. (1991): 'Binary distributive quantifiers'. WCCFL 10, Stanford

Linguistics Student Association, Stanford University, Stanford. (1994): 'Together and alone'. Ms. UCLA, Los Angeles. (to appear): Parts and Wholes in Semantics. Oxford UP, Oxford.

Schwarzschild, R. (1994): 'Plurals, presuppositions, and the sources of distributivity'. Natural Language Semantics 2.

Simons, P. (1987): Parts. A Study in Ontology. Oxford, Clarendon Press. 